\title{
Guest Editorial: Special Issue on Nature Inspired Cooperative Strategies for Optimization (Part I)
}

\author{
Germán Terrazas • Carlos Cruz • Juan R. González
}

Received: 10 August 2011 / Accepted: 16 August 2011 / Published online: 30 August 2011

(C) Springer-Verlag 2011

Nature is an unlimited source of resilient, robust, fine tuned, complex mechanisms and phenomena which have been the subject of exploration and exploitation in science and engineering. In particular, understanding complex cooperative systems in nature has driven computer scientists and practitioners to outstanding developments ranging from versatile models and architectures to highly effective methodologies and computing strategies. Examples of these include, but are not limited to, artificial bee colony, memetic-computing agent-models, bacteria metabolism inspired robot controllers, evolutionary design optimization, particle swarm optimization, emergent collective robotics behavior to name but a few.

This thematic issue brings a selection of the best research works published in the IV International Workshop on Nature Inspired Cooperative Strategies for Optimization (NICSO) held on May 2010 in Granada, Spain. The aim of NICSO is to bring together scientists from all over the world to discuss the latest ideas and state of the art on nature inspired cooperative strategies. As in its previous editions, NICSO 2010 has covered topics related to adaptive behavior, ant colonies, amorphous computing, artificial life, bio-inspired architectures, distributed computing, evolutionary robotics, evolvable systems, membrane computing, software self-assembly, evolutionary computation, swarm intelligence and quantum

G. Terrazas $(\varangle)$

School of Computer Science, University of Nottingham,

Nottingham, UK

e-mail: gzt@cs.nott.ac.uk

C. Cruz · J. R. González

Department of Computer Science and Artificial Intelligence, University of Granada, Granada, Spain

e-mail: carloscruz@decsai.ugr.es

J. R. González

e-mail: jrgonzalez@ decsai.ugr.es computing. Since NICSO 2010 has been one of the most successful editions to date we chose to split this thematic issue in two parts. The first part focuses on swarm intelligence, evolutionary design optimization, distributed memetic computing models and neural networks design. The second part centers on robotics research bringing together strategies for self-organization, control architecture design and emergent collective behavior.

This collection of papers starts with the work of K. Diwold, A. Aderhold, A. Scheidler and M. Middendorf who present a performance analysis of Artificial Bee Colony (ABC) Optimization where some new selection schemes are also introduced. The proposed ABCs are compared against Particle Swarm Optimization (PSO), two forms of the hierarchical PSO, the differential evolution algorithm, and Ant Colony Optimization on six standard benchmark functions: Ackley, Griewank, Rastrigin, Rosenbrock, Sphere and Schaffer. The results lead to interesting guidelines for the configuration of the ABC parameters: the population size; the time limit to abandon solutions that do not improve in favor of random ones; and the number of onlooker bees. It is also seen that $\mathrm{ABC}$ is not as robust as it was previously thought, and that the parameters have to be tuned properly for a successful optimization. Finally, the comparison with other algorithms showed that the best performing variant of ABC is also statistically equivalent or better than the other tested algorithms on all the benchmark functions with the exception of Griewank for which the hierarchical PSOs was better.

The second paper by P. Novoa-Hernández, C. C. Corona and D. A. Pelta brings a novel variant of multi-swarm PSO for tackling adaptation issues in multi-modal dynamic optimization problems (DOP). In particular, they argue that both diversity loss and outdated memory are important problems to be addressed when applying PSO to DOP. The authors develop two strategies for generating diversity in dynamic 
environments, and dealing with convergence as well as low quality swarms. Inspired by the multi-swarm quantum PSO, the approach applied to the diversity generation is based on partitioning the swarm subject to the quality of the particles. The strategy for dealing with convergence and low quality swarms focuses on applying adaptive fuzzy rules which classify the swarm according to its degree of diversity (low or high) and the level of fitness (bad or good). These procedures are then combined and applied to benchmark problems such as the Moving Peaks Benchmark and others with different landscape, the experimental results of which reveal significant improvements.

M. Farnsworth, E. Benkhelifa, A. Tiwari, M. Zhu and N. Moniri present a framework for multi-objective design optimization of Micro-Electro-Mechanical systems (MEMS). The authors provide a combination of computer aided design tools and soft computing optimization algorithms with the aim to facilitate an environment in which designers automate modelling, simulation and optimization at all levels of MEMS design process. The novelty of this approach lays on coupling simulation and analysis tools for MEMS design with multi-objective evolutionary algorithms NSGA-II and MOGA-II. Five different design case studies of increasing complexity are used to test the framework: a meandering spring, a meandering resonator, a folded flexure resonator, an ADXL150 accelerometer and a micro gyroscope. The obtained results are shown to be competitive and sometimes superior in comparison to the ones achieved by previous research works. Moreover, the authors provide a comparative study on the performance of NSGA-II and MOGA-II on MEMS problems demonstrating that MOGAII provides better results for most of the selected case studies.

J. E. Amaya, C. Cotta and A. J. Fernández-Leiva contribute with a collaborative distributed memetic computing model. The methodology presented in this paper consists on employing architectures of memetic agents for tackling hard combinatorial optimization problems. The authors propose three different topologies of interaction between agents, each of these endowed with solving strategies such as hill climbing, tabu search, memetic algorithm and genetic algorithm. As a proof of concept, both homogeneous and heterogeneous instances of this cooperative model are applied to the Tool Switching Problem. The obtained results reveal that such collaborative memetic approach performs better than the current state of the art methodologies. Nonetheless, it is also shown that the proposed schema is generic and adaptive, hence paving the way with an appealing strategy for addressing other types of combinatorial optimization problems.

Finally, J. F. Miller and G. M. Khan report on a neurons inspired strategy to model artificial neural networks. In particular, authors identify and discuss fundamental properties seen in biological neural systems which could be incorporated in artificial neural networks (ANNs) design, thus giving origin to what they coin developmental artificial neural networks (DANNs). This work focuses on a novel approach for addressing catastrophic forgetting, one of the crucial weakness of most ANNs models. Authors argue that current solutions to such problem ignore key aspects needed to model information processing observed in the biological brain. Thus, the model presented here is based on evolving programs to continuously modify networks which develop over multiple problem instances without losing the ability to solve previously encountered problems. The presented strategy employs Cartesian Genetic Programming Developmental Network (CGPDN), a graph-based form of genetic programming that idealizes the behavior of a neuron in terms of seven main processes. In order to validate this approach, experiments show the co-evolution of two agents against a minimax based checkers program and evolved CGPDN checkers player against a minimax based checkers program.

We consider that the content presented in this issue is a compilation of high quality research product of several months of coordinated work between authors, reviewers and editors. We thank authors for their utmost effort on preparing and submitting their manuscripts and also reviewers for their support and assistance on the strict peer review process each contribution has been subjected to. As guest editors, we hope the articles published in this thematic issue are perceived as key contributions to the field of cooperative strategies for optimization and propel the development of innovative research related to this topic. 